\title{
Local Atomic Structure of a Clean Surface by Surface Extended X-Ray Absorption Fine Structure: Amorphized Si
}

\author{
F. Comin, ${ }^{(a)}$ L. Incoccia, ${ }^{(a)}$ P. Lagarde, ${ }^{(b)}$ G. Rossi, ${ }^{(b)}$ and P. H. Citrin \\ AT\&T Bell Laboratories, Murray Hill, New Jersey 07974
}

(Received 12 October 1984)

\begin{abstract}
The application of near-edge, surface, extended x-ray absorption fine structure to the study of a clean surface is reported. Direct evidence is found for surface recrystallization of ion-damaged (amorphized) $\mathrm{Si}$, whereas no such evidence is seen for evaporated (amorphous) Si. The procedures described here are applicable to almost all clean or adsorbate-covered surfaces.
\end{abstract}

PACS numbers: $68.20 .+\mathrm{t}, 61.40 .-\mathrm{a}, 61.80 . \mathrm{Jh}, 78.70 . \mathrm{Dm}$

We report the first study of local geometric structure from a clean surface using near-edge, surface, extended $x$-ray absorption fine structure (NEXAFS, SEXAFS). The present application of these techniques is a marked departure from the study of adsorbates ${ }^{1-4}$ and the use of the adatom's characteristic x-ray absorption to obtain surface selectivity. Here, Auger electrons with low escape depth are used to isolate the contribution of the adsorbate-free surface atoms. The system chosen for study is ion-bombarded (amorphized) $\mathrm{Si}$. We show that while the local structure of bulkamorphized $\mathrm{Si}$ is essentially indistinguishable from that of bulk-evaporated (amorphous) $\mathrm{Si}$, their surfaces are very different. In fact, the local structure of the amorphized Si surface closely resembles that of the bulk crystal, i.e., the nominally amorphized surface is actually locally ordered. These unexpected findings have direct implications on the characterization of amorphous materials and on the processes of amorphization and crystal regrowth. In addition, our results demonstrate the general applicability of SEXAFS and NEXAFS to the study of surface structure either in the presence or absence of an adsorbate species.

Bulk EXAFS data from clean single-crystal $\mathrm{Si}$ and from in situ e-beam evaporated $\mathrm{Si}$, both at room temperature, were obtained by monitoring the $\mathrm{Si} K L L$ Auger-electron yield under UHV conditions $\left(\leq 10^{-10}\right.$ torr) with equipment previously described ${ }^{1,4}$ [see Fig 1(a)]. The $K$-edge features up to $\sim 90 \mathrm{eV}$ above threshold are more clearly displayed in Fig. 1(b) by means of standard background-subtraction and $k$-space conversion procedures. ${ }^{1,5}$ Comparison of these spectra from systems with very different medium-rangeorder (MRO, i.e., at the second- and third-neighbor level) shows corresponding differences extending even near the edge, where multiple scattering can be important. Since the range of these data lies between the regimes of NEXAFS $(\leq 30 \mathrm{eV})$ and SEXAFS (typically $\geq 50-400 \mathrm{eV}$ ), a formal single-scattering EXAFS analysis is not applicable for extracting absolute phase shifts and amplitudes. ${ }^{5}$ However, such information is unnecessary here for the comparison of relative degrees of MRO. In particular, Fourier transforming
(FT) the data in Fig. 1(b), shown in Fig. 2(a), gives clear evidence of strong second- and third-shell contributions in the case of crystalline $\mathrm{Si}$, whereas only a very weak corresponding structure beyond the first coordination shell is present in the case of amorphous $\mathrm{Si}$. Aside from trivial broadening of the FT peaks, our lower- $k$ data show the same relative intensity change in medium-range structure as that observed in previous higher- $k$ EXAFS data from crystalline and amorphous semiconductors. ${ }^{5-7}$ In fact, because the medium-range second- and third-shell FT peaks become strongly damped at higher $k$ as a result of Debye-Waller-type effects, ${ }^{5-7}$ their relative intensity here is an even more sensitive probe of the MRO.

In Fig. 2(b) we show the FT of data taken by monitoring either the $\mathrm{Si} K L L$ electron yield or the total electron yield (TY) from clean room-temperature single-crystal samples which were amorphized (sputtered) with saturation doses $\left(>10^{17}\right.$ ions $\left./ \mathrm{cm}^{2}\right)$ of 2 $\mathrm{kV} \mathrm{Kr}{ }^{+}$or $\mathrm{Ne}^{+}$ions. The TY is composed mainly of low-energy secondaries $<20 \mathrm{eV}$ whose escape depths are $>50 \AA$, so that the TY detection mode is inherently not surface sensitive. ${ }^{8}$ Accordingly, the crystalline region well beyond $50 \AA$ (where the rare-gas

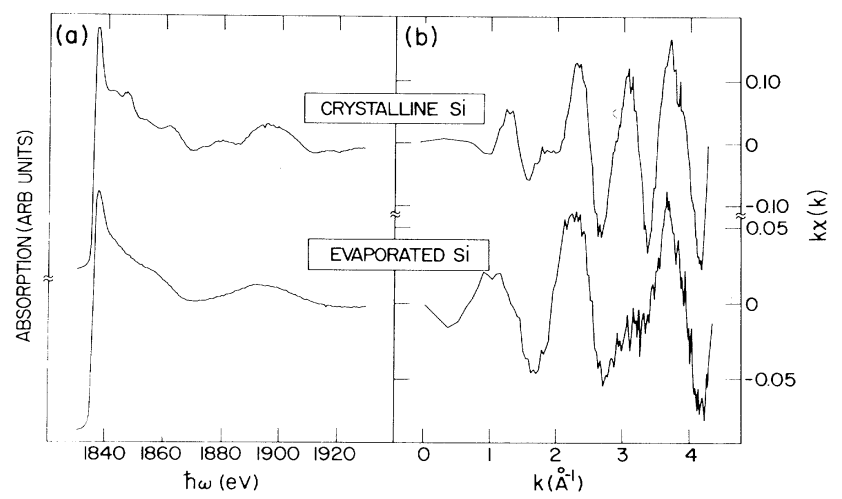

FIG. 1. (a) $K$-edge absorption spectra from single-crystal and evaporated $\mathrm{Si}$ measured with $\mathrm{Si} K L L$ Auger electrons. (b) Corresponding data after background subtraction and conversion into $k$ space. 


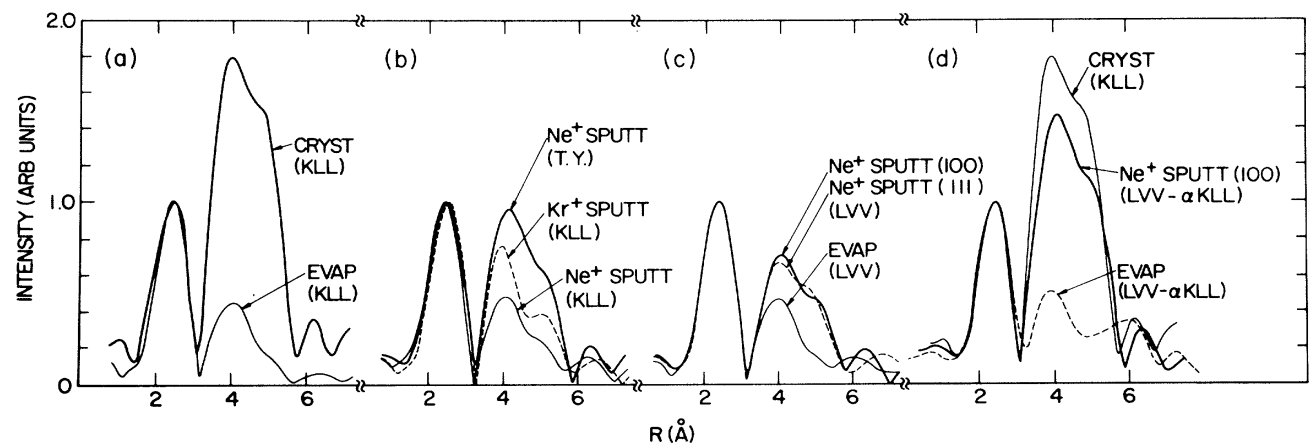

FIG. 2. Fourier transforms of background-subtracted data as a function of Si sample preparation and measurement detection mode. Intensities of the first-coordination-shell peak at $\sim 2 \AA$ have been normalized for all data (they varied $< \pm 10 \%$ ). Intensity variations of unresolved second- and third-coordination-shell structure at $\sim 4-5 \AA$ reflect changes in medium-range order. The first-, second-, and third-shell peaks correspond to actual Si distances of $2.35,3.84$, and $4.50 \AA$, respectively. (a) Single-crystal vs evaporated Si measured with $K L L$ Auger electrons (raw data shown in Fig. 1). (b) $\mathrm{Ne}^{+}$-sputtered vs $\mathrm{Kr}^{+}$. sputtered $\mathrm{Si}$ measured with $K L L$ Auger electrons vs total electron yield $\left(\mathrm{Kr}^{+}\right.$-sputtered TY data, not shown, are identical to $\mathrm{Ne}^{+}$-sputtered TY). (c) $\mathrm{Ne}^{+}$-sputtered $\mathrm{Si}(100)$ or $\mathrm{Si}(111)$ vs evaporated $\mathrm{Si}$ measured with $L V V$ Auger electrons [raw $\mathrm{Ne}^{+}$sputtered $\mathrm{Si}(100)$ data shown in upper half of Fig. 4]. (d) Bold line, Fourier transform of raw difference spectrum for $\mathrm{Ne}^{+}-$ sputtered $\mathrm{Si}$, shown as a bold curve in the lower half of Fig. 4; dashed line, same procedure applied to evaporated $\mathrm{Si}$; light line, data from single-crystal $\mathrm{Si}$, reproduced from panel (a) for comparison.

ions have not penetrated ${ }^{9}$ ) is found to contribute substantially to the TY absorption spectrum. The $K L L$ $\left({ }^{1} D\right)$ Auger electrons at $\sim 1615 \mathrm{eV}$ probe less deeply into the bulk because of their $\sim 25$ - $\AA$ escape depth. ${ }^{10}$ As expected, these electrons sample more of the iondamaged region, so that the second- and third-shell structure in the $K L L$ FT data is weaker than that obtained in the TY mode. Also observed is the difference in the FT data between the $\mathrm{Kr}^{+}$- and $\mathrm{Ne}^{+}$sputtered samples due to the different penetration depths of these ions. ${ }^{9}$ A pictorial view of the damaging depths induced by the $\mathrm{Kr}^{+}$and $\mathrm{Ne}^{+}$ions compared with the approximate sampling depths of the TY and $K L L$ Auger-electron detection modes is shown in Fig. 3. Note that with $K L L$ electrons the $\mathrm{Ne}^{+}$sputtered sample [Fig. 2(b)] exhibits features very similar to those of the evaporated one [Fig. 2(a)], i.e., the MRO in the damaged region of amorphized $\mathrm{Si}$ closely resembles that of evaporated $a-\mathrm{Si}$, again as expected.

Having shown that the degree of MRO can be readily monitored in the FT data and that its variation with depth can be probed with different detection schemes, we now apply these methods to the study of a clean surface. Recall that conventional SEXAFS or NEXAFS experiments with adsorbed species ${ }^{1-4}$ exploit one of the major characteristics of the absorption process, namely, its atom specificity. Such adatom experiments are inherently surface selective, and the choice of detection mode, i.e., Auger, TY, or partial yield, is governed only by considerations of optimizing signalto-background ratio or discriminating against unwanted photoemission. For the study of clean surfaces, on the other hand, the choice of detection modes is critical for one to distinguish between the surface and bulk contributions. The essential idea here is to measure two Auger transitions, both resulting from the initial core-electron vacancy but each with very different electron escape depth. In the case of Si these are the $L V V$ and $K L L$ electrons, produced by sequential decay of the original $K$-shell core hole, i.e., $1 s$ photoemission $\rightarrow K L L$ Auger decay $\rightarrow L V V$ Auger decay. The more surface-sensitive $L V V$ electrons can be made even

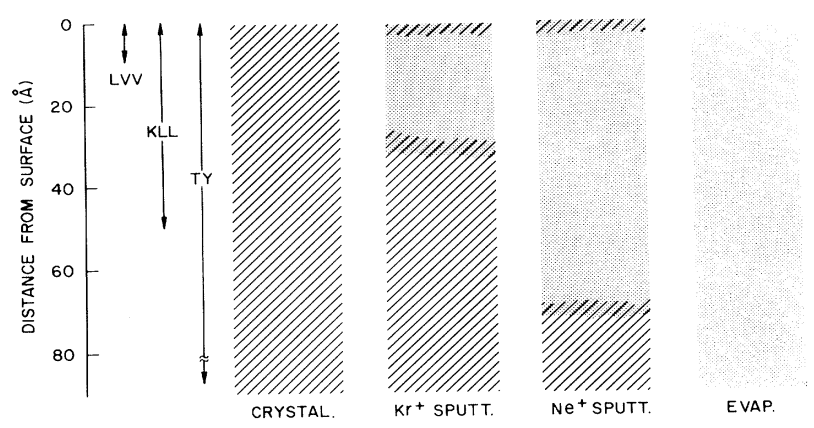

FIG. 3. Pictorial view of four different Si samples-single crystal, $\mathrm{Kr}^{+}$sputtered, $\mathrm{Ne}^{+}$sputtered, and evaporated - showing approximate depth profiles of crystalline and amorphous regions in each. Varying proportions of these regions are probed depending on the sample and the detection mode: Total electron yield (TY) is least surface sensitive, $\mathrm{Si}$ $K L L$ Auger yield is more sensitive, and $\mathrm{Si} L V V$ Auger yield is most sensitive. Note that the total probing depths for the different modes are larger than the corresponding electron escape depths. 
more so by use of $s$ polarized light, shallow takeoff collection angles, and an energy resolution comparable to the natural width of the Auger transition (to minimize detection of inelastically scattered electrons). In the present experiments the effective escape depth ${ }^{10}$ for the $\sim 90-\mathrm{eV} L V V$ electrons ${ }^{11}$ is $\sim 5 \AA$, or about 5 times smaller than that for the $\sim 1615-\mathrm{eV} K L L$ electrons.

The upper half of Fig. 4 directly compares the background-subtracted $K$-edge data obtained with the $K L L$ and $L V V$ detection modes from the same $\mathrm{Ne}^{+}$. sputtered Si sample. The more surface-sensitive $L V V$ data exhibit additional structure which the FT data, shown in Fig. 2(c), clearly identify as increased MRO.

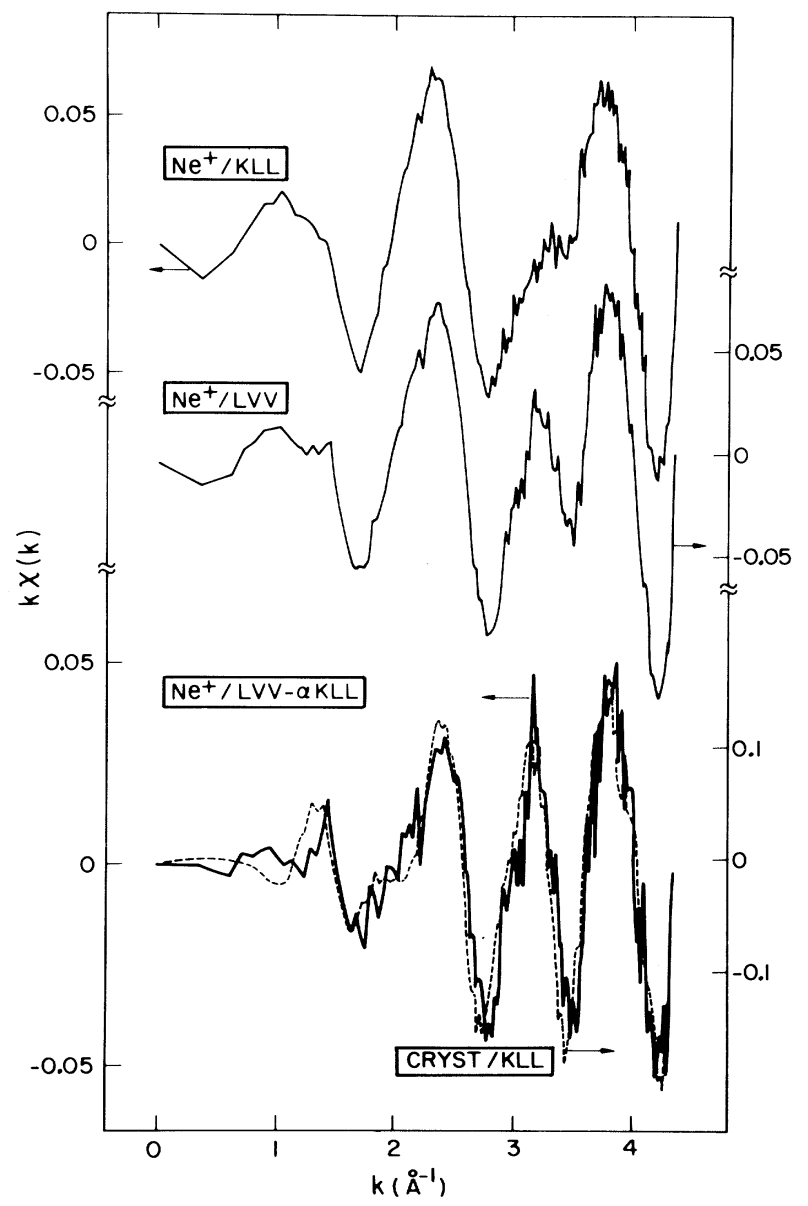

FIG. 4. Upper half, background-subtracted data from $\mathrm{Ne}^{+}$-sputtered $\mathrm{Si}$ measured with $K L L$ and $L V V$ Auger electrons; lower half, bold curve is the difference between data measured with $L V V$ electrons and an amount $\alpha$ of data measured with $K L L$ electrons. $\alpha$ is the ratio of fractional surface contributions in the two data sets and is 0.8 , accounting for the overall reduction in scale (left). Dashed curve is the single-crystal data reproduced from Fig. 1(b) for comparison.
Note that this surface crystallinity is independent of the initial single-crystal orientation and that it is decidedly absent in the evaporated $\mathrm{Si}$. Note also that the surface contribution to the total $L V V$ data is only fractional since the amorphized region beneath the surface layer is still sampled by the $L V V$ electrons. It is, however, straightforward for one to separate the surface contribution from the bulk by taking linear combinations of the $L V V$ and $K L L$ data (such procedures are common with use of photoemission data of different depth sensitivities ${ }^{12}$ ). The result of the isolation of the approximately one-monolayer-thick surface layer by subtraction of the appropriately weighted $K L L$ from $L V V$ data $^{13}$ is shown as a bold curve in the lower half of Fig. 4; superposed on it as a dashed curve are the single-crystal data from Fig. 1(b). The striking similarity between these raw data, as well as the comparison between their FT data in Fig. 2(d), makes clear the high degree of surface crystallinity in the amorphized sample. The same difference procedures for evaporated Si [see Fig. 2(d)] again show no such effect.

These surprising results have a number of implications. The small penetration depths of the ions and the saturation doses used both imply that a process of surface recrystallization originates from and on top of the bulk-amorphized $\mathrm{Si}$. That is, the observed surface crystallinity is not a result of insufficient damage to the initially crystalline surface but is, instead, a process which is stimulated by the ions that created the amorphized region (see Fig. 3). Furthermore, the equilibrium tendency to minimize the number of dangling bonds, taking advantage of the additional degrees of freedom available at the surface, is insufficient to account for the reordering since the evaporated Si surface does not exhibit such behavior. While the metastable structure from the quenched evaporated Si may play a role in the difference between the evaporated and ion-bombarded surfaces, the role played by the ion radiation thermal spike (i.e., localized annealing) seems likely to be even more important. The methods described here allow future work to address these and related questions by study of the surface versus bulk local ordering and recrystallization as a function of initial sample preparation (e.g., e-beam evaporation, sputter deposition, glow discharge, laser quenching), sample temperature, and subsequent ion bombardment. Such structural studies should provide surfacesensitive short-range information unobtainable with any other probe.

The significance of these findings extends beyond its applicability to $\mathrm{Si}$ and amorphous systems. Our methods demonstrate that NEXAFS and SEXAFS, in general, can be meaningfully applied to clean surfaces. The lack of requiring long-range order and the wide availability of other elements with both low- and 
high-electron-escape-depth Auger transitions allow these techniques to be applied to almost any substrate. Since the atom-specific photoabsorption is unaffected by the presence of adsorbates, questions relating to not only clean surface reconstruction but also to surface relaxation induced by chemisorption can therefore be studied. In short, a complete local characterization of adsorbate and substrate surface atom structure before and after chemisorption should be possible. For these future experiments to be most productive, optimized surface sensitivity and more fully extended data above threshold will be required.

To summarize our results, the techniques of SEXAFS and NEXAFS we previously used for determining local geometries of adsorbates on ordered single crystals have been extended to the study of surfaces lacking both order and adsorbates. With these methods a novel recrystallization has been identified in the surface layer of clean amorphized Si. Considerable generalization to studies of local surface structure should now be possible.

The authors thank W. L. Brown, L. C. Feldman, J. C. Phillips, and I. K. Robinson for helpful conversations and comments about the manuscript. The work done at the Stanford Synchrotron Radiation Laboratory was supported by the U. S. Department of Energy, Office of Basic Energy Sciences.

(a) Permanent address: Istituto di Struttura della Materia del Consiglio Nazionale delle Ricerche, I-00044 Frascati, Italy.

(b) Permanent address: Laboratoire pour l'Utilisation du Rayonnement Electromagnétique, Université de Paris-Sud, F-91405 Orsay, France.
1P. H. Citrin, P. Eisenberger, and R. C. Hewitt, Phys. Rev. Lett. 41, 309 (1978), and 45, 1948 (1980).

${ }^{2}$ L. Johansson and J. Stöhr, Phys. Rev. Lett. 43, 1882 (1979).

3J. Stöhr, K. Baberschke, R. Jaeger, R. Treichler, and S. Brennan, Phys. Rev. Lett. 47, 381 (1981).

${ }^{4}$ P. H. Citrin, J. E. Rowe, and P. Eisenberger, Phys. Rev. B 28, 2299 (1983).

5P. A. Lee, P. H. Citrin, P. Eisenberger, and B. M. Kincaid, Rev. Mod. Phys. 53, 769 (1981), and references therein.

${ }^{6}$ F. Evangelisti, M. G. Proietti, A. Balzarotti, F. Comin, and L. Incoccia, Solid State Commun. 37, 413 (1981).

${ }^{7}$ E. A. Stern, C. E. Bouldin, B. von Roedern, and J. Azoulay, Phys. Rev. B 27, 6557 (1983).

8Partial yield detection of even low-escape-depth secondaries is also not surface sensitive because the majority of primary photoelectrons originate from within the bulk.

${ }^{9}$ J. Lindhard, M. Scharff, and H. E. Schiott, K. Dan. Vidensk. Selsk. Mat. Fys. Medd. 33, No. 14 (1963); in Si the average ranges of $2-\mathrm{kV} \mathrm{Kr}^{+}$and $\mathrm{Ne}^{+}$ions are 32 and $68 \AA$, respectively.

${ }^{10}$ Our use of low effective takeoff angles and $s$-polarized light offsets our choice of low-energy resolution, giving essentially the theoretical escape depths for $\mathrm{Si}$; cf. M. P. Seah and W. A. Dench, Interface Anal. 1, 2 (1979). Further details will be described elsewhere.

${ }^{11}$ The $1 \mathrm{~s}$ photopeak sweeps through the $90-\mathrm{eV}$ analyzer window, accounting for the truncation of the $L V V$ data and (for consistent comparison) the $K L L$ data at this particular energy.

12P. H. Citrin, G. K. Wertheim, and Y. Baer, Phys. Rev. Lett. 41, 1425 (1978), and Phys. Rev. B 27, 3160 (1983).

${ }^{13}$ The determined weighting coefficient $\alpha$ of 0.8 in Figs. 2 (d) and 4 assumes an effective layer thickness of $1.5 \AA$, a value intermediate between $\frac{1}{2}(0.78+2.35)=1.57 \AA$ for the $\mathrm{Si}(111)$ planes and $1.35 \AA$ for the $\mathrm{Si}(100)$ planes. The exact value is not critical, however, since our results are insensitive to $\alpha$ within $\pm 15 \%$. 